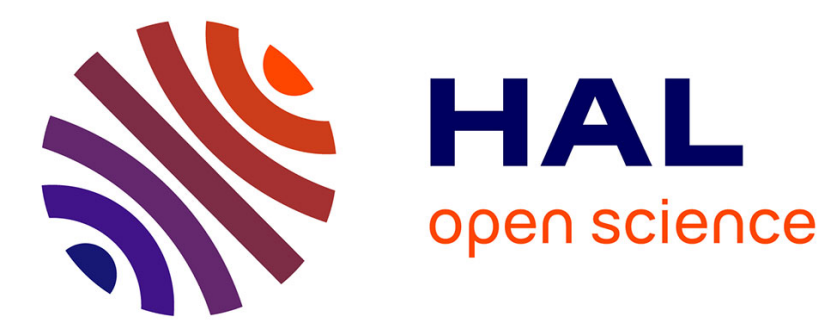

\title{
Semantic search using modular ontology learning and case-based reasoning
}

Nesrine Ben Mustapha, Hajer Baazaoui, Marie-Aude Aufaure, Henda Ben Ghezala

\section{- To cite this version:}

Nesrine Ben Mustapha, Hajer Baazaoui, Marie-Aude Aufaure, Henda Ben Ghezala. Semantic search using modular ontology learning and case-based reasoning. the 2010 EDBT/ICDT Workshops, Mar 2010, New York, United States. pp.301-312. hal-00831707

\section{HAL Id: hal-00831707 \\ https://hal-centralesupelec.archives-ouvertes.fr/hal-00831707}

Submitted on 7 Jun 2013

HAL is a multi-disciplinary open access archive for the deposit and dissemination of scientific research documents, whether they are published or not. The documents may come from teaching and research institutions in France or abroad, or from public or private research centers.
L'archive ouverte pluridisciplinaire HAL, est destinée au dépôt et à la diffusion de documents scientifiques de niveau recherche, publiés ou non, émanant des établissements d'enseignement et de recherche français ou étrangers, des laboratoires publics ou privés. 


\section{Semantic Search using Modular Ontology Learning and Case-Based Reasoning}

\author{
Nesrine Ben \\ Mustapha \\ Laboratoire RIADI \\ ENSI Campus \\ Universitaire de la \\ Manouba 2010 \\ nesrine.benmustapha \\ @riadi.rnu.tn
}

\author{
Hajer Baazaoui \\ Zghal \\ Laboratoire RIADI \\ ENSI Campus \\ Universitaire de la \\ Manouba 2010 \\ hajer.baazaouizghal \\ @riadi.rnu.tn
}

\author{
Marie-Aude \\ Aufaure \\ Ecole Centrale Paris, \\ Laboratoire MAS \\ Chaire SAP Business \\ Objects Grande Voie \\ des Vignes 92295 \\ Chatenay-Malabre \\ marie- \\ aude.aufaure@ecp.fr
}

\author{
Henda ben \\ Ghezala \\ Laboratoire RIADI \\ ENSI Campus \\ Universitaire de la \\ Manouba 2010 \\ Henda.benghezala \\ @riadi.rnu.tn
}

\begin{abstract}
In this paper, we present a semantic search approach based on Case-based reasoning and modular Ontology learning. A case is defined by a set of similar queries associated with its relevant results. The case base is used for ontology learning and for contextualizing the search process. Modular ontologies are designed to be used for case representation and indexing. Our work aims at improving ontology-based information retrieval by the integration of the traditional information retrieval process, the use of ontology learning (OL) and the Case-Based Reasoning (CBR) process. In fact, the proposed approach uses the CBR with semantic Web language markup -by ontology- for case representation and indexing. Ontology-based similarity is used to retrieve similar cases and to provide end users with alternative documents recommendations. The main contribution of this work is the use of a CBR mechanism and an ontological representation for two purposes: Resource Retrieval from Web and ontology learning and enrichment from cases. This approach builds a knowledge corpus - represented by ontology modules - resulting from the collaboration actions of users. The experiment shows an improvement in terms of results' precision and ontology learning relevance.
\end{abstract}

\section{Categories and Subject Descriptors}

I.2.4 [Knowledge Representation Formalisms and Methods]Representation languages

\section{General Terms}

Design, Theory.

\section{Keywords}

Semantic Web, ontology, modular ontology, semantic search, information retrieval, Case-Based Reasoning.

\section{INTRODUCTION}

Over the past few years and with the continuous and rapid growth of Web information volume, information access and knowledge management has become challenging. Thus, adding a semantic dimension to the Web, with ontology deployment, contributes to solve many problems in many domains (Information retrieval, knowledge sharing, communication between Web agents, etc.). In fact, in the context of the semantic Web [12], the need for using domain ontology in information retrieval (IR) has been explored by many approaches to better answer users' queries [7, 15, 26, 28]; the explicit representation of knowledge is made through ontologies that are an explicit specification and a formal shared conceptualization [18]. Ontologies have contributed to the emergence of semantic search engines. They are used to enhance query formulation, document indexing and conceptual classification of results. In fact, one challenging issue for developing successful semantic search engine is the availability of ontologies used for the contextualization of user queries.

Context representation and formalization for enhancing retrieval process are the main research challenges when designing and developing content-based search engines. Current studies in this area $[14,24,14]$ focus on the integration of different methods and techniques derived from artificial intelligence and knowledge management domains (Ontology, similarity measures, semantic distances, intelligent retrieval methods) in order to outperform the relevance of search results.

Since intelligent retrieval is one of the main application of CaseBased Reasoning paradigm (CBR) [1, 25], semantic formalization in CBR systems has also become an increased research area [4, 13, 19, 24, 31]. In CBR systems, semantics are the main source of reasoning, similarity calculation and case adaptation.

Our ongoing work aims at the developing multi-domain CBRBased search system able to handle ontology learning and perform ontology-based information retrieval. In this paper, we describe main approaches related to ontology learning for semantic search engine and the use of CBR approach for information retrieval in Section 2. CBR-based search can be improved by integrating ontology. Then, the rest of paper is organized as follows. In Section 3, we present the semantic search approach based on a multi-layer ontology warehouse, CBR mechanism, an enhanced search process and ontology learning. In Section 4, use cases are presented to illustrate the proposed approach. Finally, evaluation test results related to ontology learning and search results are presented. Finally, we conclude the paper and outline the directions of our future work in Section 5.

\section{RELATED WORK}

In this section, we present the related work on the following topics:

Integration of ontology learning in semantic search framework and ontology learning from Web

Case-Based Reasoning for IR process and Ontology for CBR mechanism. 


\subsection{Integration of ontology learning in semantic search}

Ontology learning (OL) aims at building ontology from knowledge sources using a set of machine learning techniques and knowledge acquisition methods. OL from Web is a specific case of OL from texts and has been widely used in the community of engineering knowledge since texts are semantically richer than the other data source type. These approaches are generally based on the use of textual corpora. The corpus should be representative of the domain used to build ontology. By applying a set of text mining techniques, a granular ontology is enriched with concepts and relationships discovered from textual data. In these approaches, human intervention is required to validate the relevance of learned concepts and relationships. In the last decade, with the enormous growth of Web information, the Web has become an important source of information for knowledge acquisition: due to its huge size and heterogeneity. This has been the cause of the appearance of many categories of OL approaches: ontology learning from textual content of the Web, ontology learning from online Web ontologies, from web dictionary and from Web heterogeneous sources. OL from Web documents require the same techniques as those used for ontology extraction from texts. Several approaches are based on eliminating tags from documents to obtain plain texts to which traditional text mining texts could be applied. These approaches are dedicated to ontology building from Web and are based on the generation of taxonomies without using a priori knowledge or natural language processing techniques and on the use of large corpus or thesaurus. In [37], an incremental approach of ontology learning from Web is proposed. In [37], a study of several types of available Web search engines and how they can be used to assist the learning process (searching web resources and compute IR measures) is described. The proposed learning process is based on four steps. The first one is a Taxonomic learning step where the user starts by specifying keywords used as a seed for the learning process using a Web search engine, the output of this step is a one-level taxonomy, a set of verbs appearing in the same context as extracted concepts. Secondly, non taxonomic relations learning are carried out. The verbs and keywords lists are used as bootstrap for building domain related patterns as well as for defining query addressed to a search engine. The third step is the recursive learning task where the two previous learning stages are recursively executed for each discovered concept. Finally, the post-processing step consists in refining and evaluating the obtained ontology. This approach is domain independent and incremental. In the same context, our previous work [5] has proposed an incremental approach of ontology learning from Web. We combined many text mining techniques and use an ontology-based IR System to classify Web documents. Our experiments have shown that ontology enrichment from document resulting from ontology based-search system is more accurate and that the relevance of search results is improved. So, our objective is to integrate ontology learning within a multi-domain search engine.

Approaches related to ontology-based information retrieval [23, 40] propose a generic framework that followed by more 7 items:

\section{Creation and management of ontologies}

Query expansion

Documents annotation

\section{Documents indexing \\ Results filtering \\ Ontology retrieval \\ Association retrieval}

The model described in [23] integrates four main modules:

Ontology Management Module (OntoMan) which includes the automated construction of ontologies from text where the user role is to specify new materials to enhance or build new ontologies.

$\square \quad$ Ontology directory

$\square \quad$ Ontology crawler.

The query engine for ontology search

In [40], the author proposes a model of generic search engines that includes all types of research (document retrieval, ontology search and association discovery). These approaches have not leaded yet to the realization of related frameworks for the following reasons:

Ontology learning from Web documents depends on the relevance of these documents related to the domain and the applied techniques

managing many domain ontologies makes the task related to the detection of user query 's context very difficult.

The next section describes how case-based reasoning can be a solution to contextualize the user search query and to outperform search results.

\subsection{Case-Base Reasoning for Information Retrieval and Ontology for CBR mechanism}

Query formulation is becoming a challenging problem. Good query formulation must include all necessary features to retrieve the relevant information, which is not an obvious task especially for the first attempt [30]. Thus, the use of an iterative process of trial and error is necessary to improve query formulation. For this reason, adaption and learning have long been viewed as crucial parts of IR systems [35]. Therefore, using a Case-Based reasoning (CBR) model in an IR process is an ambitious area. CBR is a problem-solving method [1]. It is based on the concept of "case" which consists of a problem description and its solution. A new problem is solved by retrieving and reusing similar problems from the "case base". If revision indicates that a new solution has to be provided for the new problem, both problems are then retained as new cases that are added to the case base for eventual retrieval and reuse [30]. The main idea under CBR consists in storing experiences as cases and problem-solving processes as instances of cases. When a new problem is encountered, the system uses the relevant past stored cases to interpret or to solve it [30]. The system performance, increases with the growth of the stored cases. In [32], many approaches have been investigated in using CBR model to overcome classic IR problems.

Given that CBR is based on retrieving similar cases by using similarity measures between terms, it is obvious that semantic knowledge can ameliorate the results of this process. In the same context, the idea of combining ontology (domain knowledge) with CBR-based systems for knowledge management has been dealt by many approaches [27] [11] [18]. In the same context, the Knowledge Sifter (KS) framework of a collaborative semantic 
search [41] creates a repository of user queries and artifacts which are produced during the retrieval process. A case-based framework is proposed for $\mathrm{KS}$ in order to recommend query specifications and refinements basing on the previously-stored user-query cases. A user query case is only generated when a user provides a relevance feedback from the query results. The user feedback is the user's evaluation of the relevance degree of a result to the refined query. This relevance feedback can be also considered as a user rating of the results information quality.

Using CBR mechanism in an ontology-based IR system could be an ambitious area in order to improve semantic indexing and query reformulation in a question-answer service [39], for improving recommendations results [3] and obtaining good precision of search results [22].

Therefore, our underlying hypothesis is that case-based reasoning supported by ontology technology is a promising approach for achieving semantic-aware search and ontology learning. Our objective is to develop a semantic search approach based on the use of many domain ontologies in order to meet any user's need. In fact, our motivation is to use past and current queries to improve the precision of provided results to next users and to index Web documents using submitted queries and discovered ontology elements from these documents. We describe in the next section the proposed approach which is composed of an enhanced semantic search approach based on case-based modular ontology [10]. We propose to combine a CBR mechanism with multidomain modular ontologies which are classified by topic in order to ameliorate query formulation, dynamic semantic indexing and eventually, the results precision. We illustrate our work with use cases and then present the evaluation tests results.

\section{SEMANTIC SEARCH APPROACH USING CASE-BASED REASONING AND MODULAR ONTOLOGY LEARNING}

In this section, we propose a generic approach allowing any search engine to develop its semantic layer from the associations between queries and documents results. Taking account of the associations between past queries and selected results to store situations search and learning from past situations to satisfy new queries of users justify the choice of using reasoning based on cases. The main components of the proposed approach are (figure 1):

An iterative content-based search process which uses a multilayer ontology-warehouse for indexing Web resources and domain cases;

\section{$\square \quad$ A CBR mechanism : Case-based Modular Ontology; \\ $\square \quad$ Ontology learning and enrichment.}

The idea behind this approach is (1) to use the previously discovered ontology-aided semantic metadata representation in OWL, and (2) to provide the user about resource characteristics and (3) to answer queries using ranked cases. At each step, a ranked set of recommended queries related to previous similar cases is provided. After case selection, new documents are imported and classified using relevance feedback [33]. The use of this technique is recommended to extend user query by taking benefit of previous user satisfaction. In this work, we propose to extend user query with previous document related to selected case. This process narrows down results and incrementally eliminates cases which are proven to be irrelevant and enrich the case base with new ones. Modular ontologies used for the domain case representation and indexing are also incrementally enriched using text mining techniques. These ontologies are designed according to a previous work [8] where a multi-layer ontological warehouse was designed to annotate discovered resources. Moreover, modular ontologies used for case representation and indexing are also enriched incrementally by using ontology learning techniques applied to textual result related to a case.

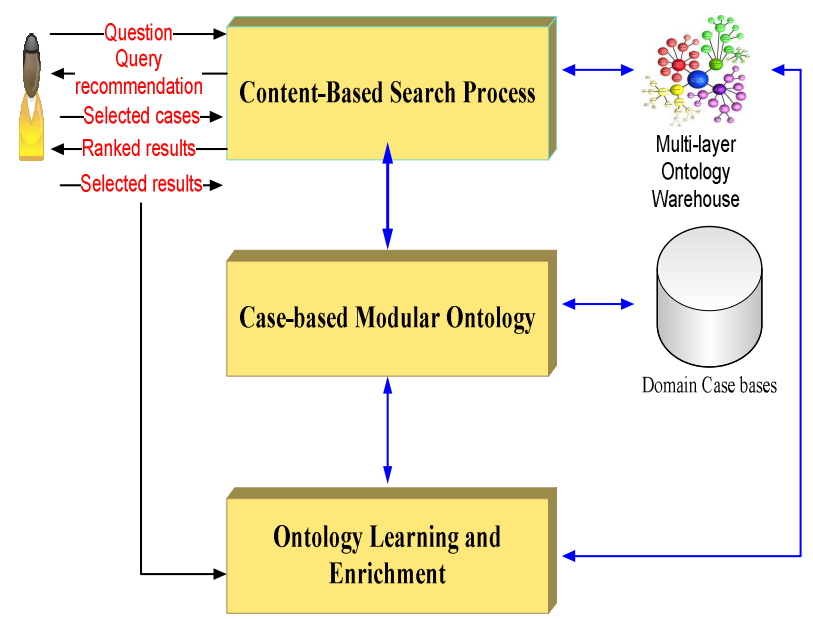

Figure1. Multi-Domain Content-Based Search based on casebased modular ontology and Ontology learning.

In the following, we describe the multi-layer ontology warehouse used for Web documents and case indexing. Then, case representation and CBR mechanism are widely explained.

\subsection{Multi-layer Ontology Warehouse for Indexing Cases}

Multi-domain ontology design is a crucial point because of the problem of ambiguity, semantic heterogeneity and concept sharing between different domains. We have proposed in [8], a multi-layer ontology warehouse designed for multi-ontologybased semantic search systems. Given that a search query contains the keyword "BMI". This term refers to three concepts related to different domains (Table 1). According to this observation, we notice that a term can be associated to many senses (represented by concepts). Each concept identifies a unique sense, plays a role in many homogeneous topics and is characterized by semantic relations with other features and concepts (this set represents its contextual signature generally used in the task of term disambiguation). A contextual signature (or Topic signature) is defined as a technique used in the area of text mining and ontology learning. A topic signature of the concept "conference" is a set of concepts that permanently occurs with this concept in the most sentences extracted from the Web documents related to this concept. For this reason, we have designed the following multi layer ontology warehouse (Figure 2).The first layer represents the topics following an ontological classification of topics regardless to the used language. Topic ontology is a set of topics connected with different types of relations. 
Table 1. Illustration

\begin{tabular}{|l|l|l|l|}
\hline $\begin{array}{c}\text { Terms, } \\
\text { language }\end{array}$ & \multicolumn{1}{|c|}{ Concept } & \multicolumn{1}{|c|}{ Topics } & \multicolumn{1}{|c|}{$\begin{array}{c}\text { Contextual } \\
\text { signature }\end{array}$} \\
\hline $\begin{array}{l}\text { BMI, } \\
\text { English }\end{array}$ & $\begin{array}{l}\text { body mass } \\
\text { index }\end{array}$ & $\begin{array}{l}\text { Medicine, } \\
\text { health, natural } \\
\text { sciences }\end{array}$ & $\begin{array}{l}\text { Health, } \\
\text { measurement, } \\
\text { nutrition balance, } \\
\text { high BMI }\end{array}$ \\
\hline $\begin{array}{l}\text { BMI, } \\
\text { English }\end{array}$ & $\begin{array}{l}\text { British } \\
\text { Midland } \\
\text { Airways }\end{array}$ & $\begin{array}{l}\text { Airways, } \\
\text { aviation }\end{array}$ & $\begin{array}{l}\text { Civil aviation, } \\
\text { united kingdom, } \\
\text { British } \\
\text { Mediterranean } \\
\text { Airways }\end{array}$ \\
\hline BMI, & $\begin{array}{l}\text { Organism } \\
\text { English } \\
\text { license fees } \\
\text { on behalf of } \\
\text { songwriters } \\
\text { and } \\
\text { composers }\end{array}$ & Music & $\begin{array}{l}\text { Songwriters, } \\
\text { composers, } \\
\text { downloaded } \\
\text { records }\end{array}$ \\
\hline
\end{tabular}

Each topic is discovered from the categories provided by most of the traditional Web search engines ("yahoo", MSN, Dmoz.). Each topic (i.e. "sport", "music", "travel", "news",..) becomes a "domain" when it is associated with a modular domain ontology. The second layer represents a set of modular domain ontologies (Odi) defined as a network of ontology modules (Mdi). An ontology Module $(M d i)$ is seen as a fragment of the domain ontology which consists of a main concept $(\mathrm{Ci})$ with its common properties (relations with others concepts $C i j$ ). Properties of a concept are defined as the most frequent relations that characterize this concept and that are used in query interfaces and relevant Web documents. Then, a module is a set of concepts strongly related by semantic relations used to identify the context of search. It is possible, therefore, to structure a user query by a conceptual graph $\mathrm{b}$ with concepts belonging to a same module. An example of an ontology module related to the domain of "computer sciences" consists of:

the main concept: "conference"

a set of related concepts that represent properties of the conference concept (topics, sponsors, proceeding, location, submission date, chair, organizing committee, etc.)

When the ontology module is extracted from textual content according to the proposed approach, properties of the main concept are obtained from the contextual signature of the concept "conference". Besides, a given Module Mdi could be in relation with other modules and could belong to several ontologies. For example, the module having as main concept "conference" could be present in several domain ontologies such as "computer science", "physics", "mathematical", etc. Given that the proposed approach of the semantic search is based on many domain ontologies, a concept cannot be only identified by a term because of the problem of ambiguity. For example, the term "table" can refer to the concept of "table of database or "a piece of furniture". For this reason, we need an identifier "Id" associated to each concept (example, "table\#1" that denotes the first sense of "table" according to Wordnet [43]). Moreover, as we have above mentioned, concepts that make up a module are extracted from the Web documents by applying text mining techniques. Therefore, erroneous concept can be discovered. The attribute "state" is used to abstract state of the concept. Only validated concepts are used in query reformulation and documents classification. The degree of credibility of a concept indicates if the discovered concept is used in the main steps of the search process These information are important in both the ontology learning and the enrichment step, and are automatically updated according to the approach proposed in $[5,6]$. The information is split into the first two layers by using the "owl: imports" constructor for linking multiple OWL ontologies to form a larger OWL ontology.

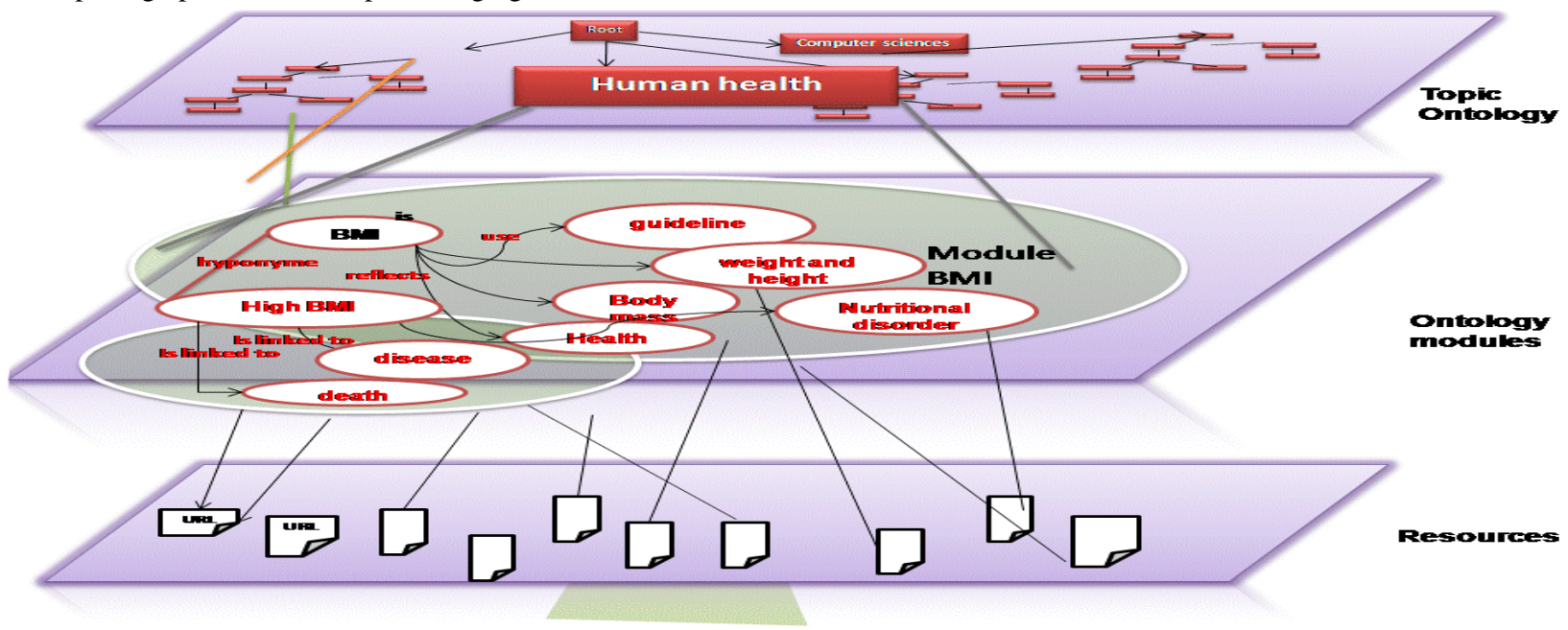

Figure 2. Multi-layer ontology warehouse for semantic search 


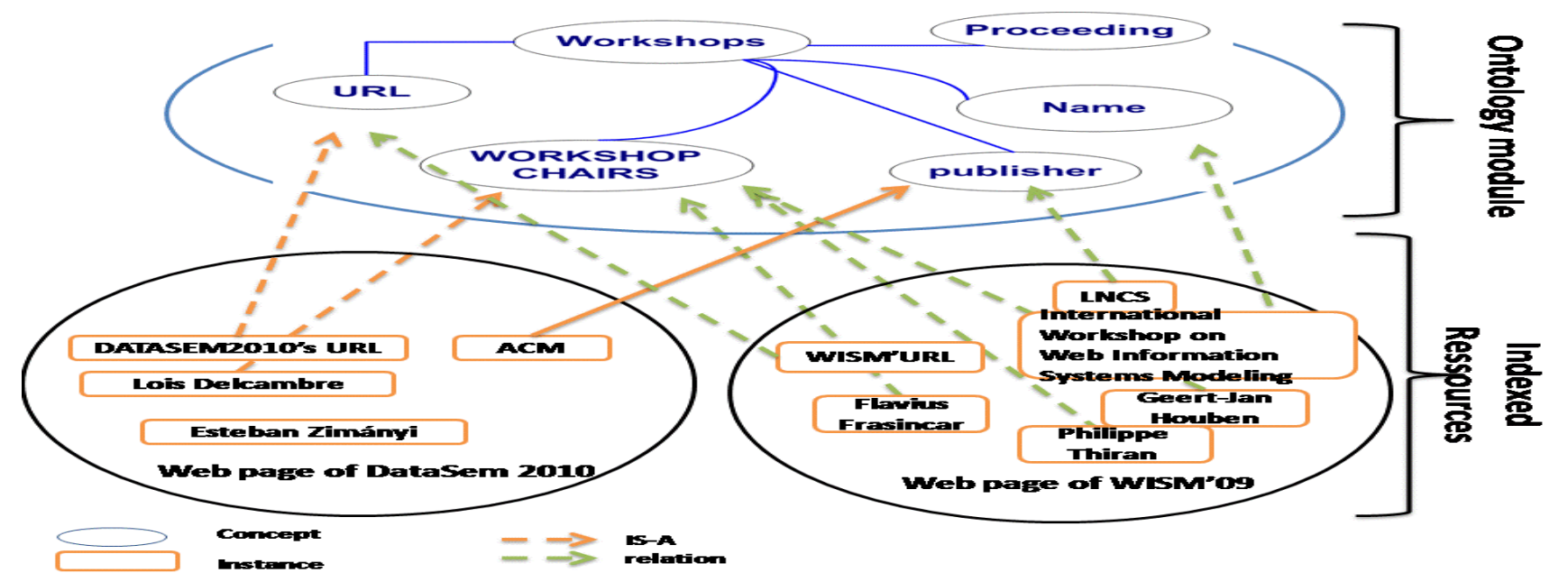

Figure 3. Relation between resources and the second layer

A concept is represented by the tuple (id, \{(ti, language, context)\} $i=1 . . n$, state, credibility Degree) where:

$\square \quad I d$ : a concept identifier associated to a sense regardless of the terminological labels and the language referencing it;

$\{(t i \text {, language, context })\}_{i}$ : a set of the triple $(t$, language, context) where $t$ is a nominal phrase referencing the concept in a target language and used in a specific context which can be the topic representing the concept role in a specific domain;

State: the state of the discovered concept. A discovered concept from text could be identified as "new candidate", "validated", "rejected", "average candidate";

Credibility degree: a degree of the concepts' accuracy according to their module.

The third layer includes the indexed resources and cases linked to ontology modules. This layer doesn't belong to the ontological layers but it is made up with resources (Web document, document fragment, a delivered software) associated with the metadata that is generated by the populated ontology module with values in order to make difference from the other resources which are indexed by the same ontology module. Then, the populated ontology module is an instance of the second layer. For example, the Web page of the workshop "datasem2010" and the Web page related to "International Workshop on Web Information Systems Modeling" are two resources indexed by the same module related to "workshops" (figure 3).

In this paper, the proposed multi-layer ontology warehouse is used for context representation with ontologies that are incrementally enriched from relevant results to queries' context. It is built by applying text mining techniques in order to discover new ontology elements from users' selected Web documents. The topic ontology is extracted from Dmoz categories. The modular ontologies are built from textual content of the Web documents. The first two layers are linked as following: each concept of topic ontology is associated to a pointer to an OWL file that represents domain ontology. Each domain ontology is built by using the "owl: imports" constructor that imports related ontology module (the second layer). Ontology modules are automatically extracted and validated in an incremental way. When a user selects new documents that are relevant to his query, the ontology module is maintained. Besides, the state of the "discovered concepts" is also updated according to the process described in [5].

\subsection{CBR Mechanism}

The main goal of this approach is to provide users with documents references lists or with fragment of documents relevant to their queries. The two main advantages of using CBR to tackle the problem are:

The ability to use and build on past experiences of users' information requests

The ability to incorporate context information.

The modular ontology is used to perform context representation with ontologies that are incrementally enriched from relevant results to queries' context. Besides, CBR is used to improve the following tasks (figure 4): Query Formulation, Query reformulation, Document classification and ranking, User Evaluation.

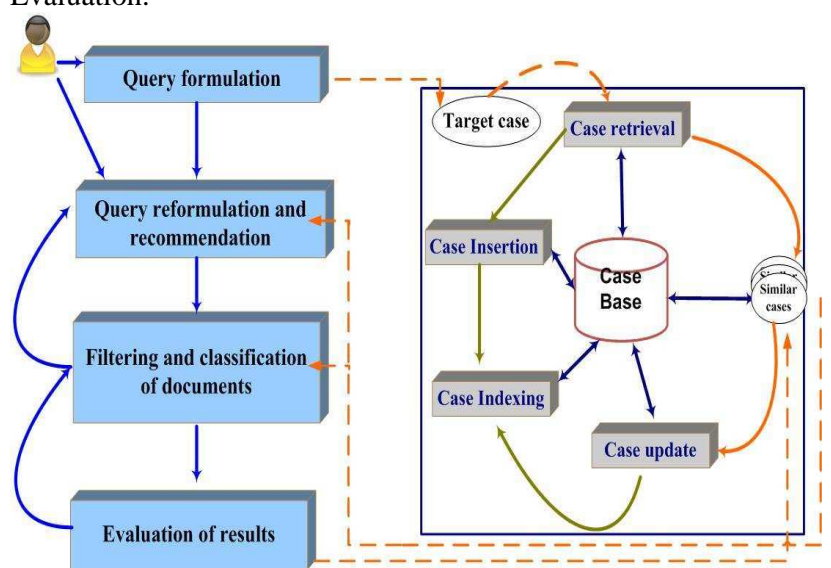

Figure 4. CBR mechanism for semantic search

In order to take into consideration all the purposes cited above, we have defined the structure of a case according to the information needed for each of the tasks cited below. 


\subsubsection{Case Representation}

A case is generally structured into three parts: a problem, a solution and a rating evaluation. The composition of these parts (figure 5) is presented and described in the following.

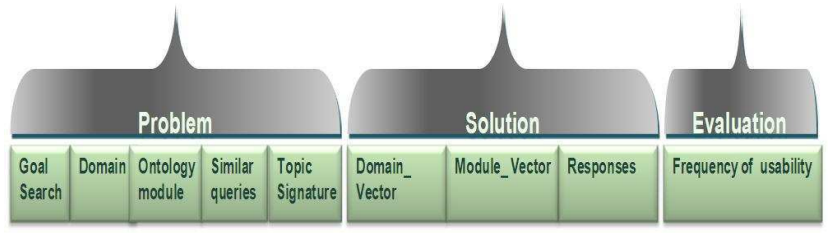

Figure 5. Case Representation.

\subsubsection{Problem Representation}

A problem consists of the description of a user need of information search. In classic IR systems based on CBR, a problem is a keyword-based query and solutions are documents URLs [44]. In the proposed approach, we describe the context of the query by the following elements which represent the context of the user needs (i.e. goal search, the topic of his search, the ontological representation (Ont_M) from his query, similar queries submitted by the user to reformulate his query and the topic signature. (Figure 5):

Goal search $(\boldsymbol{G S})$ : The type of goal search considering the classification presented in the work related to open information retrieval [34].

$\square$ Domain $(\boldsymbol{D})$ : The Domain or Topic of a search. It represents a concept in the topic ontology.

Ontology module (Ont_M): An instantiated graph of concepts related to a particular ontology module that represents the common structure of a set of similar queries.

$\square \quad$ Similar queries $\left(Q \_s e t\right)$ : A set of similar term-based queries related to the ontology module.

Topic signature (TopicSign): The Topic signature related to concepts belonging to the ontology module Ont_M. The topic signatures are context vectors built for concepts [2].

When a user submits a query related to the domain of computer sciences in order to search international workshops related to the topic of Web engineering, a new problem is specified using the features that have above mentioned. This problem is then described by the n-uplet (GS= navigational, $\mathbf{D}=$ "computer sciences,

Ont_M

"http://www.SemSearch.com/computer_sciences/ontology\#works hop.owl" (Figure 3), Q_set $=$ \{("workshops + web engineering), (Workshop + semantic Web engineering), (international workshop on Web) $\}$, TopicSign $=\{$ "Web, semantic Web, Web design, submission deadline, paper, author"].

Furthermore, ontology module and similar query are used to retrieve similar problems to a new query that can be formulated by user as keyword-based query, natural language query or formbased query. Topic signature is a set of concepts that have not yet been validated to be linked to the ontology module but are used in the query formulation.

\subsubsection{Solution Representation}

In classic IR systems based on CBR, the solution is specified as the set of URLs of relevant Web documents. These systems are generally based on a limited collection of documents. In the context of the Web, it is not obvious to provide the users only with previously selected documents. For this reason, a vector of weighted concepts called "module_vector" is constructed to obtain the most relevant documents. Given that the documents containing terms referring to the concepts defined by the ontology module could not be relevant to the domain of the query, we propose to eliminate the documents whose representative vectors are dissimilar with a second vector called "Domain_Vector".

In this work, the solution is generally composed of pointers to the set of resources described by the problem metadata. It contains also other new elements needed for new imported Web documents filtering and classification with the use of Relevance Feedback (RF). A solution is a set of answers to a submitted question (problem) and two conceptual vectors. If the search goal of the user is navigational, the solution is then a set of Web documents. But, if the user searches for a definite response to a question (i.e. informational goal search), the answer may be a concept or an instance of concepts. In this paper, we focus on the navigational search. For this reason, examples in Section 4, solutions look like just sets of URL's. The solution section is specified by the following elements:

Module_Vector: This vector is the n-uple $\left(\left(W_{1} C_{1}\right) W_{2} C_{2}\right)$ $\left.\left.W_{3} C_{4}\right), \ldots,\left(W_{m} C_{m}\right)\right) . W_{i}$ represents the weight of the terms $t_{i}$ referring to concepts defining the ontological module Ont_M. " $m$ " is the number of concepts of the ontology module. This vector will be used not only to rank documents that are imported from external sources as the Web but also to learn more similar documents and to index them with the appropriate case.

Domain_Vector: This vector is the n-uple $\left(\left(W_{1} C_{1}\right) W_{2} C_{2}\right)$ $\left.W_{3} C_{4}\right), \ldots,\left(W_{n} C_{n}\right)$. Wi represents the weight of all terms referring the concepts that define the domain " $D$ " to which belongs the ontology module Ont_M. This vector can be used to decrease the noise of others imported documents which are not relevant to the domain " $D$ ".

Response (Set_response): The research results whose relevance to this case requests is indicated by the user. The results can be Web pages selected by the users, concepts or text fragments. Their type depends on the Goal Search (GS).

We recall that the relevance of document in the vector-based model [36] is calculated by a cosine similarity function between two vectors. According to this model, if the similarity value is negative then, the document is not relevant to a query otherwise, it is relevant. When a given recommended case is selected by the user to refine his query, the two vectors are respectively used. Use of these vectors aims at extending the query with weighted concepts in order to obtain a new vector-based query and to eliminate from results the set of documents containing the query keywords but that do not match with the appropriate domain. An example of a solution of a case is presented in table 1.

\subsubsection{Evaluation Representation}

This bloc is made of some information such as a timestamp corresponding to the case creation, use accounts and contexts in which they apply. In the following section, the CBR mechanism is described.

\subsubsection{CBR cycle}

The architecture of CBR is composed of four parts: case indexing, case retrieval, case update and case insertion (figure 3 ).

\subsubsection{Case Indexing}

The power of the CBR system relies on its ability to find the more relevant cases from case base accurately. For this reason, case 
indexing is very important to retrieve related and useful case for query reformulation, display case recommendations and import external similar Web documents. The multi-layer ontology warehouse is used to index cases by the means of an ontology module. In fact, the case indexing consists in referencing each case by the ontology module which is very important to find similar cases from a huge case base. We remind that an ontology module is composed of a main concept which is relevant to the search domain and of other concepts that represent features characterizing this main concept. Then, a case is indexed by an OWL file representing an instantiated ontology module.

\subsubsection{Case Retrieval}

Once the case representation and indexing are completed, a retrieval structure can be organized. Similar case retrieval is released by the main tasks of information retrieval: query submission, query modification by the user, choosing a recommended case by the user. According to case representation defined in the previous section, there are many level of similarity starting from identity to nearly similarity. It depends on the number of common features of the problem presentation between a target case " $C_{t}$ " and a source case " $C_{s}$ " (extracted from the case base). Many case retrieval technologies exist such as nearest neighbor method, inductive method (decision Tree) and knowledge-guided method or a combination of these three methods. In our proposition, we used the nearest neighbor one [29].

When a user submits a query and selects the domain of his search from the topic ontology, two alternatives are possible. In the first one, the submitted query is related to a new ontology module that does not exist in the ontology warehouse. This circumstance is similar to a classic IR iteration where a new case is inserted and an ontology module is constructed from the selected Web documents. In the second alternative, the ontology module that specifies the query is recognized, and then cases "Cs" indexed by this module "Ont_M" are extracted from the case base. The first list is ranked according to the shared concepts between the queries "Set_Q" of Ct and Cs. These cases are called "strongly similar". A second list is made up by cases indexed by the nearest ontology modules to "Ont_M" of "Ct". The extracted cases are ranked according to the number of relation paths that exist between "Ont_M" and the other ontology modules indexing cases "Cs". These cases are called "nearly similar". This process is described by the following algorithm:

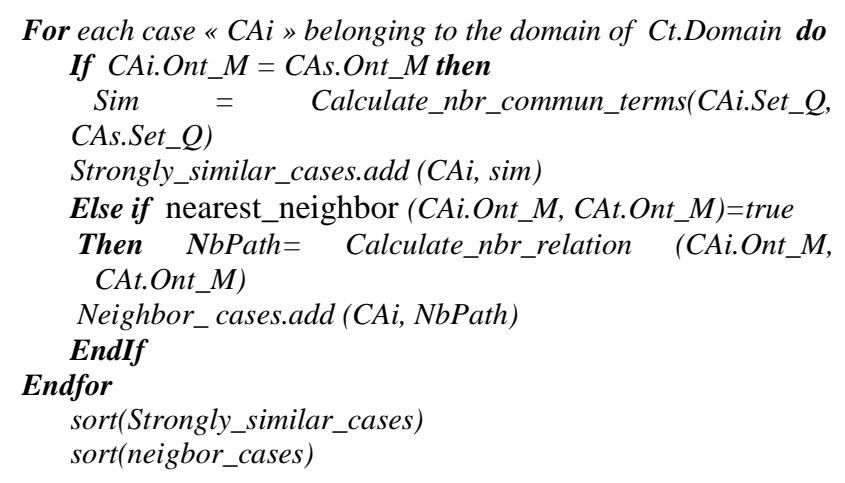

\subsubsection{Case Insertion}

After submitting a query, if no similar cases exist in the case base, a new case is automatically inserted into the case base. This case comprises the features describing the user query and is indexed by a new ontology module extracted from the selected documents. These features are:

Goal of search, domain and a set of queries used in the step of query reformulation

Conceptual graph related to the request of search,

Topic signature which involves concepts appearing frequently with the concepts of "Ont_M" in the same sentences extracted from the selected Web documents from user ("Set_response")

After inserting the problem, the insertion of the solution of the case can be made only after the validation of the choice of Web documents by the user. After that, the construction of the two vectors is done as following:

The construction of the vector ("Module_Vector") of concepts involves the calculation of weight of each term referring the concepts of the ontology module "Ont_M" using vector model and ontology.

The construction of the vector ("Domain_Vector") of concepts is the result of calculating the weight of each term referring all the concepts of the domain ontology (a network of ontology modules) using the vector model.

The weighting procedure is released with CF-ICF measure which gives approximately the relevance of concept to a given document. A CF/ICF (concept frequency / inversed concept frequency) approach has been developed to adapt at the concept level the classical TF/IDF method which operates at the term level [36]. The used formula is:

$$
W i j=\frac{W i j}{\sum[W i j]^{2}}
$$

Where $\mathrm{Wij}=(1+\log \mathrm{CFij}) * \mathrm{ICF}$ where $\mathrm{CF}$ is "Concept Frequency" which is proportional to the frequency of occurrence of all terms referring a given concept and ICF is the Inverse Concept Frequency.

$$
\mathrm{ICF}=\log \left(\frac{N}{f i}\right)
$$

Where $\mathrm{N}$ : total Number of concepts of ontology module or domain ontology, depending on which vector is calculated.

\subsubsection{Case update}

This procedure is the adaptation phase in a process of reasoning over cases. Once an existing case in the base case is selected, its enrichment remains an important phase. This update consists of:

The insertion of new documents selected by other users

The enrichment of the conceptual graph of query with further discovered concepts and relations from these documents.

The update of topic signature, the set of responses and the computation of the two vectors.

\subsection{Enhancing Content-Based Search Process}

The proposed IR is an iterative process that uses CBR mechanism in the following tasks (figure 3): Query Formulation, Query reformulation, Document classification and ranking, User Evaluation and Ontology enrichment. 


\subsubsection{Query Formulation}

At the first step, the user can formulate a query in three ways:

Keyword-based query which is a set of words proposed by the user to define his request. It is similar to queries submitted in the traditional IR systems.

Natural language question which is written by the user and it is a syntactically well-defined question.

Form type query: this last kind of formulation is used when the user navigates in the domain ontology and selects the appropriate concepts to formulate the question, then, the main concept and properties will be shown in a form to be filled by the user.

The related domain ontology is provided to help users to navigate through the ontology module. The user then submits a query or selects the appropriate ontology module. The user-provided search query is analyzed to identify the appropriate ontology module defined in the multilayer modular ontology. WORDNET is used to recognize and to disambiguate new concepts. The ontology module is updated and assigned with a sense identifier so that the next user doesn't need to select the appropriate senses of query's terms. In Wordnet, each term is associated with a set of Senses. Knowing the sense of a concept eases not merely its enrichment from Wordnet but also the disambiguation of the term-based query. Hence, senses are needed to distinguish between the senses of a term. We refer to a sense by a number associated to the sense of a term from Wordnet. When the ontology module is recognized, a search form is displayed with others features to be filled by the user. Then, similar cases are extracted from case base and query's recommendations are displayed with its results sorted by ranking score (section 3.2.2.2). If the user selects one of the recommended cases, a procedure of query reformulation is released.

\subsubsection{Query reformulation}

Query reformulation is done by the system basing on the selected similar cases. The query is represented by the aggregation of the weighted concepts of "module vector" with the terms of the active query. The weight attributed to the active query is "1"; then the final query vector is obtained by calculating the average of weights of concepts of the two vectors. An adapted case is constructed. A new set of queries is formed by adding userprovided features and topic signature to queries "Set_Q" described by the selected cases. If there are no similar cases, the query is refined by adding synonyms. A set of adapted queries are sent to the search engine and a set of Web documents are delivered.

\subsubsection{Document classification and ranking}

This step consists of two tasks: document filtering and document ranking. Document filtering is done when the number of cases indexed by related domain ontology is important, and ontology module belongs to many different topics (like the ontology module related to the concept "conference" which is present in many topics such as medicine, computer sciences, music, ..). The domain vector including weighted domain concepts is used to calculate the cosine between the conceptual vector model of returned documents and the domain vector ("Domain_Vector") of the selected case. Documents whose cosine is negative are eliminated. In order to rank the most relevant documents, the vector ("Module_Vector") is updated by adding user-provided terms which should have a higher weight than the others concepts.
Each document is represented by a vector $D j=(d 1 j, d 2 j, \ldots .$. , Dnj) where "dij" is the weight of this concept in the document $\mathrm{Dj}$ and $\mathbf{n}$ represents the number of concepts of the vector. The query is represented by the adapted ("Domain_Vector") $\mathrm{Q}$ ' $=\left(\mathrm{q}_{1}, \mathrm{q}_{2} \ldots\right.$, $\mathrm{q}_{\mathrm{m}}$ ) where qi is the weight of concepts in this vector. The weights of concepts are calculated using the formula CF-ICF (defined in the section 3.2.2.2). The measure of similarity between documents and the query is calculated with the cosine formula:

$$
\operatorname{SIM}\left(\mathrm{Dj}, \mathrm{Q}^{\prime}\right)=\frac{\sum_{i=1}^{N} d_{i j} q_{i}}{\sqrt{\sum_{i=1}^{N} d_{j i}^{2} \bullet \sum_{i=1}^{N} q_{i}^{2}}}
$$

Once the similarity is calculated, user documents with strictly positive similarity are displayed.

\subsection{Incremental Ontology Enrichment}

The final task of this system is the enrichment of the ontology module indexing the updated case or a new inserted case from the Web documents making up the solution of this case. Each document will be the input of the ontology learning phase of the process proposed in a previous work [5,6]. Text mining techniques (syntactic patterns and verb based patterns) are used to discover new concepts and new relations between the concepts of the ontology module and the topic signature. It is possible to extract new terms which have not a stable relation with the ontology module. In this case, these terms are added to the topic signature of the case (instead of being added to the ontology module) in order to be used in a next iteration.

\section{USE CASES DEMONSTRATION}

In this section, two use cases related to several users which respectively submit two queries $Q 1$ and $Q 2$ that appear as two independent requests, but according to domain knowledge, the results of one is the answer to the other. The query "Q1" is "What is the BMI in medicine? Q2 is "How to measure the nutritional disorder? “.

Table 2. Case Representation of C1

\begin{tabular}{|c|l|}
\hline $\begin{array}{c}\text { Case } \\
\text { Index }\end{array}$ & \multicolumn{1}{|c|}{\begin{tabular}{c}
\multicolumn{1}{c|}{ http://www.SemSearch.com/Medicine/ontology\# } \\
BMI.owl
\end{tabular}} \\
\hline \multirow{3}{*}{ Problem: } & $\begin{array}{l}\text { GS= navigational, } \\
\text { D=http://www.SemSearch.com/Medicine\# } \\
\text { Ont_M: (Is (“BMI", X?)) } \\
\text { Set_Q=(BMI, (BMI + Body Mass index),(BMI + } \\
\text { body fat measure) } \\
\text { TopicSign= weight, height, body, } \\
\text { health............. }\end{array}$ \\
\hline \multirow{3}{*}{ Solution } & $\begin{array}{l}\text { Module_Vector } \\
\text { Vector domain } \\
\text { Set_response = } \\
\text { http://en.wikipedia.org/wiki/Body_mass_index }\end{array}$ \\
\hline
\end{tabular}

We suppose that the first user submits the query "Q1". This first iteration can be done with any search engine given that there is no learning at this stage. Concerning this query, we cannot find any related ontology module in the ontology warehouse nor similar cases as the query keywords are not clear. A graph corresponding to the query is extracted using lexico-syntactic patterns. Then, the corresponding keywords are sent to a search engine. The user selects the relevant documents to be saved and a new case " $\mathrm{C} 1$ " is 
inserted in the case base (Table 2). Both the insertion of a new case and the calculation of the two vectors are automatically done. After the case insertion, the construction of the ontology module related to the concept "body mass measure" is done by fulfilling the following tasks:

Extraction of sentences containing the term "BMI" from selected Web documents associated to case $\mathrm{C} 1$ (figure 6).

Application of lexico-syntactic patterns and syntactic frames to discover new related concepts and relations.

Validation of the ontology elements discovered for the set of Web documents.

The resulting ontology module is illustrated by Figure 6 .

A second user submits the next query Q2. This second iteration is fulfilled by learning from similar queries. The difference between the two queries is that the first user exactly knows the concept for which he is searching ("BMI") while the second user only knows the role of this concept (without knowing the fact that the nutritional disorder is a factor for the BMI). Use of a traditional search engine to answer to the query Q2 will lead to the difficulty of finding that BMI is used to measure a nutritional disorder. BMI is not figured as a term in the second query. But according to the proposed approach, ontological index of cases can provide the second user with a similar case existing in the case base.

By applying this approach, we have remarked that there is a common result between the two queries. However, by using Google engine, we didn't find the same result. In fact, similar cases retrieval for query Q2 provides the most ranked recommended cases ( $\mathrm{C} 1$ and many others cases (as $\mathrm{C} 2$ shown by Table 3 ). For this second user, we can provide a summary of what is a nutritional disorder, using the ontology module (concepts in the neighborhood of nutritional disorder) and give a set of recommended documents. In this context, we supposed that a previous user submits queries related to nutritional topic. The explication of this common result is described as following; the ontology module indexing the case $\mathrm{C} 1$ is strongly related to the ontology module indexing the case $\mathrm{C} 2$ (figure 7) because $\mathrm{BMI}$ measures the percentage of body fat, it may be a useful tool to estimate a healthy body weight and a high BMI is linked to nutritional disorder. The relation between the two ontology modules was discovered in the step of ontology enrichment of the case $\mathrm{C} 1$. The two ontology modules were automatically created in the first iteration by applying text mining techniques on documents of the case $(\mathrm{C} 1)$

\section{Table 3. Case Representation of C2}

\begin{tabular}{|c|c|}
\hline $\begin{array}{l}\text { Case } \\
\text { Index }\end{array}$ & $\begin{array}{c}\text { http://www.SemSearch.com/Medicine/ontology\# } \\
\text { nutrional_problem.owl }\end{array}$ \\
\hline Problem: & $\begin{array}{l}\text { GS= navigational, } \\
\text { D=http://www.SemSearch.com/Medicine\# } \\
\text { Ont_M: (conceptual graph that include the } \\
\text { concept of nutritional disorder and all measures) } \\
\text { Set_Q= (nutritional problems measurement, } \\
\text { nutritional disorder measures ...............) } \\
\text { TopicSign= health, body, food, ........... }\end{array}$ \\
\hline Solution & $\begin{array}{l}\text { Module_Vector } \\
\text { Vector domain } \\
\text { Set_response = } \\
\text { http://www.merck.com/pubs/mmanual_ha/sec3/c } \\
\text { h17/ch17b.html }\end{array}$ \\
\hline & \\
\hline
\end{tabular}

So, BMI is one of the measurements of nutritional problem. There are others answers such as "serum albumin measurement" (C2). .There are others answers such as "serum albumin measurement" (C2). The selection of one of the proposed cases narrows the process of search. This implies the use of the vector module. In this example, we suppose that the second user selects the case C1 among the recommended cases, then, a set of queries are submitted to import others Web documents whose similarity with the vector "Module_Vector" is important. composers). Then, we apply the documents filtering by using the domain vector. We remark that majority of removed Web documents include the term BMI, but they have not the same mining of "body mass index".

\section{What is Body Mass Index (BMI)?}

$\mathrm{BMI}$ is a guideline that uses your weight and height to figure out whether you are at risk for weightrelated health problems. BMl reflects body mass instead of body fat and is used to screen for health or motritumal disurders. For an individual, other data must be used to figure out whether a high BMI is IInked to disease and death.

The relationship between fatness and BMI is influenced by age and gender. For example. women have more body fat than men at the same BMI and older people have more body fat than younger people at the same BMI.

BMI Categories

$\begin{array}{ll}\text { BMII } & \text { Weight Category } \\ \text { Less than } 18.5 & \text { Underweight } \\ 18.5-24.9 & \text { Normal Weight (healthy range) } \\ 25-29.9 & \text { Overweight } \\ 30 \text { and above } & \text { Obese }\end{array}$

\section{Limits to $B M$}

For most people. BMI provides a good measure of obesity. However. BMI leaves out a lot of relevant intormation including activity level, muscle mass, and percent body fat. It may overestimate body fat in athletes and others who have a muscular build or it may underestimate body fat in older persons and others who have lost muscle mass.

Figure 6. Example of a textual fragment used for discovering ontology elements. 


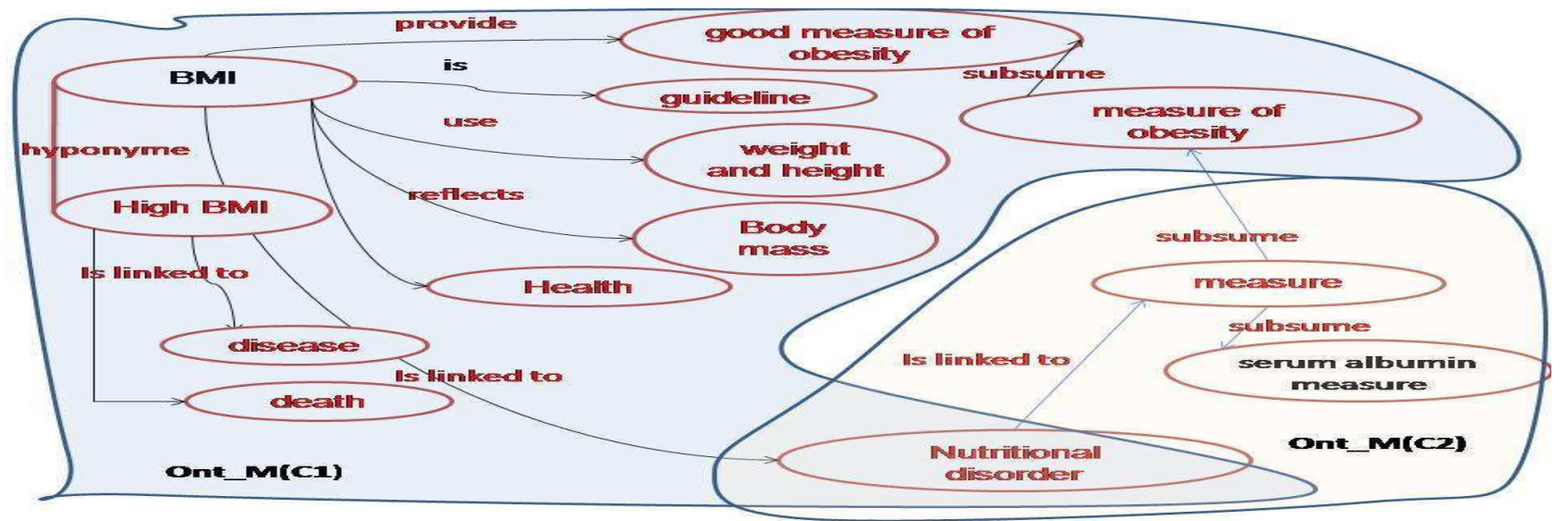

Figure 7. Relation between two ontology modules indexing respectively two similar cases.

In fact, there are other senses of BMI such as ("BMI as Commercial noun of the British Midland Airways" and BMI as Web site that collects license fees on behalf of songwriters

\section{TEST RESULTS EVALUATION}

The prototype supporting the proposed approach is developed using the service "GOOGLE_API" and the library JENA.

We used both XML-based index and an indexing scheme for the efficient storage and the retrieval of the cases. The size of a case base will be the same as the size of the ontology elements given that the cases are instances of the ontology modules. The XMLbased indexing scheme uses ontology module to index the user query cases. This leads to efficient algorithms for the associative retrieval of the relevant related cases, thereby avoiding a sequential search of the case base, as it is the case in other casebased search systems [43]. To evaluate the proposed approach, we distinguish tow main criteria:

Evaluation of the ontology learning process and comparison with our previous approach [5] ;

$\square \quad$ Evaluation of relevance of search results.

\subsection{Ontology enrichment evaluation}

In this section, two ontologies are compared. The first one is an ontology resulting from our previous approach "OntoCoSemWeb".

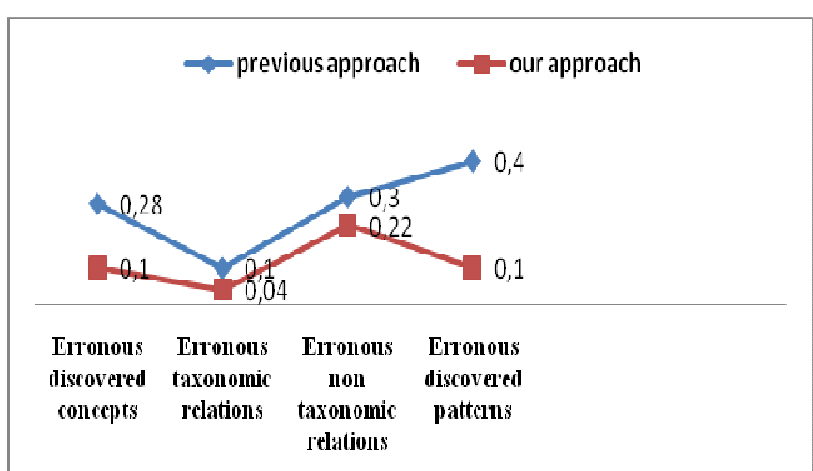

Figure. 8. Comparison of noise in learning process between our previous approach and a combined one.
This approach is based on the metaontology built upon the extraction of all textual elements from Web documents imported by a search engine. The second one is a modular ontology resulting from the approach described below, using a modified version of OntoCosemWeb [6]. The number of errors found in the discovered concepts and learned patterns has been compared (Figure 8 ). Noise in learning results was incredibly decreased by the first iteration. So, the combination of the two processes can produce more relevant Web documents from which only an ontology fragment (module) will be enriched. This has also an effect on processing time.

\subsection{Search Result Evaluation}

We defined three scenarios (Figure 9) where we compared the obtained results. The first scenario presents the results of Google search. The second scenario represents the results obtained by the proposed approach and where there are similar cases (neighbor cases as $\mathrm{C} 1$ and $\mathrm{C} 2$ ) in a case database covering 50 cases. The third scenario presents the results of similar applications where there are highly similar cases in a database containing 100 cases.

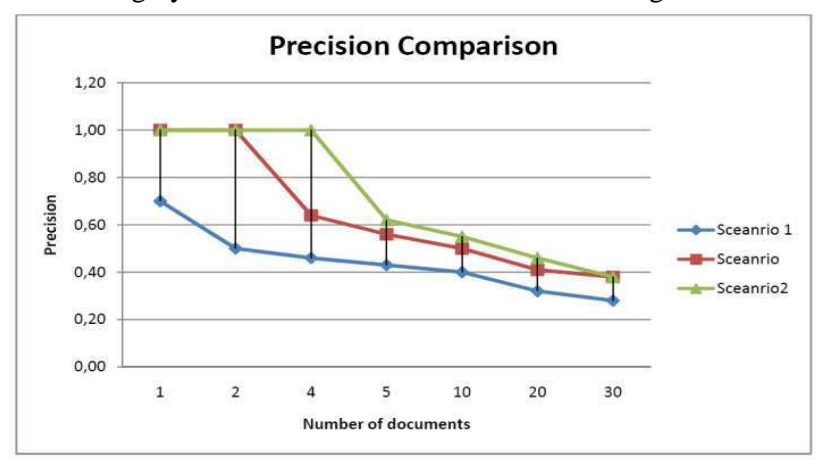

Figure 9. Precision comparison

The results have revealed that:

$\square$ the accuracy of the results was significantly improved by using CBR with modular ontologies;

the size of the case base has a considerable effect on improving the relevance of search results (scenario 2);

Strongly similar cases are important to better contextualize users searches (scénario3).

The results are shown in Figure 9. 


\section{CONCLUSION and FUTURE WORK}

The challenge addressed by this paper is to find a solution to improve the contextualization of requests based on past users' queries and the construction of ontologies from the query context (documents selected by the user). In this paper, we have presented related work of using CBR technology for IR system and Ontology learning for IR. In fact, since intelligent retrieval is one of the main applications of Case-based reasoning paradigm (CBR), semantic formalization in CBR systems has also becoming an increased research area. In CBR systems, semantics are the main source of reasoning, similarity calculation and case adaptation. Our underlying hypothesis is that case-based reasoning supported by ontology technology is a promising approach for achieving semantics aware search and ontology learning. In this work, we discussed an enhanced semantic search based on case-based modular ontology, by which the traditional information retrieval, ontology and CBR can be integrated. Our recommender approach uses Case-Based Reasoning (CBR), with semantic Web language markup (ontology) for case indexing. Modular Ontologies are used to index cases that classify Web resources. Ontology-based similarity is used to retrieve similar cases and provide the users with alternative recommendations. Case base are also used in ontology enrichment. A case study and test evaluation (with a base of 100 cases) revealed that the combination of ontology and CBR mechanism can have a positive impact on the relevance of search results.

Our ongoing work also aims at extending the evaluation when searching in large digital libraries taking into account social networks.

\section{REFERENCES}

[1] Aamodt, A., and Plaza, E. Case-based reasoning: foundational issues, methodological variations, and system approaches. AI Communications 7, 1 (1994), 39-59.

[2] Agirre, E., and Lacalle, O. L. D. Publicly available topic signatures for all wordnet nominal senses. In the 4rd International Conference on Language Resources and Evaluation (LREC) (Lisbon, Portugal, 2004).

[3] Aktas, M., Pierce, M., Fox, G., and Leake, D. A web based conversational case-based recommender system for ontology aided metadata discovery. In Proceedings of the Fifth IEEE/ACM International Workshop on Grid Computing (GRID 2004) (2004), IEEE Computer Society Press.

[4] Anders, KP., Challenges in CBR for Context Awareness in Ambient Intelligent Systems, Proceedings of the 1st Workshop on Case-based Reasoning and Context Awareness (CACOA) co-located with the 8th European Conference on Case-Based Reasoning (ECCBR) (2006), Ölüdeniz/Fethiye, Turkey.

[5] Baazaoui Zghal, H., Aufaure, MA., Ben Mustapha, N. Extraction of Ontologies from Web Pages: conceptual modeling and tourism Journal of internet Technologies, (2007).

[6] Baazaoui-Zghal, H. Aufaure, MA., Ben Mustapha, N. A Model-Driven approach of ontological components for online semantic web information retrieval", Journal on Web
Engineering, Special Issue on Engineering the Semantic Web, Rinton Press, vol. 6, n4, pp 309-336.

[7] Baziz. M. (2004)Towards a Semantic Representation of Documents by Ontology-Document Mapping. The Eleventh International Conference on Artificial Intelligence, Varna, Bulgaria, Christoph Bussler, Diete Fensel (Eds.), SpringerVerlag Germany, 33-43.

[8] Ben Mustapha N., Baazaoui Zghal H., Aufaure MA and Ben Ghezala H. (2009a), Combining Semantic Search and Ontology Learning for Incremental Web Ontology Engineering, International Workshop on Web Information Systems Modeling (WISM 2009) (Held in conjunction with CAiSE 2009), Amsterdam, 8-10 juin 2009.

[9] Ben Mustapha N., Baazaoui Zghal H., Aufaure MA and Ben Ghezala H. (2009c), Ontology learning from Web: survey and framework based on semantic search, second International conférence on Web and Information Technologies, ICWIT2009, kerkena, Tunisia, 12-14 juin, 2009.

[10] Ben Mustapha N., Baazaoui Zghal H., Aufaure MA and Ben Ghezala H. Enhancing Semantic Search using Case-Based Modular Ontology, the 25th Symposium On Applied Computing (SAC), track on "The Semantic Web and Applications" (SWA), 22 - 26 March 2010, Sierre, Switzerland

[11] Bergmann, R., and Schaaf, M. On the relations between structural case-based reasoning and ontologz-based knowledge management. In Wissensmanagement (2003), U. Reimer, A. Abecker, S. Staab, and G. Stumme, Eds., vol. 28 of LNI, GI, pp. 279-286.

[12] Berners-Lee, T., H. J., and Lassila, O. The semantic web. Scientific American (May 2001).

[13] Díaz-Agudo, B., González-Calero, P.A., An Architecture for Knowledge Intensive CBR Systems, In Blanzieri, E., Portinale, L., (Eds.): Advances in Case-Based Reasoning (Procs. of the 5th European Workshop on Case-Based Reasoning, EWCBR 2000), Lecture Notes in Artificial Intelligence, 1898, Springer, 2000.

[14] C. Doulaverakis, E. Nidelkou, A. Gounaris, Y. Kompatsiaris, An Ontology and Content Based Search Engine For Multimedia Retrieval, 10th East-European Conference on Advances in Databases and Information Systems, ADBIS 2006, Thessaloniki, Hellas, 3-7 September, 2006.

[15] D. Fensel Ontologies: a silver bullet for Knowledge Management and Electronic Commerce, Berlin,Springer Verlag.tion - Evaluation. Institut AIFB, University of Karlsruhe. 2005.

[16] Forbus, K., Birnbaum, L., Baker, J., Wagner, E., Witbrock, M., Analogy, Intelligent IR, and Knowledge Integration for Intelligence Analysis: Situation Tracking and the Whodunit problem, in: Proceedings of the 2005 International Conference on Intelligence Analysis

[17] [14] Lenz, M., Bartsch-Sporl, B., Burkhard, H., and Wess, S Case-Based Reasoning Technology - From Foundation to Applications,Lecture Notes in Artificial Intelligence 1400, Springer Verlag, 1998

[18] Gao, J., and Deng, G. The research of applying domain ontology to case-based reasoning system. In Proceedings of 
International Conference on Services Systems and Services Management (2005), pp. pp.1113 - 1117.

[19] Gómez-Gauchía, H., Díaz-Agudo, B., González-Calero, P.A., "Ontology-Driven Development of Conversational CBR Systems". In Roth-Berghofer, T.R., Göker, M.H., Güvenir, H.A., (Eds.): Advances in Case-Based Reasoning, Procs. of the 8th European Conference on Case-Based Reasoning, ECCBR 2006. Lecture Notes in Artificial Intelligence, 4106, Springer, 2006

[20] Gruber T.: Toward principles for the design of ontologies used for knowledge sharing. International Journal of HumanComputer Studies, special issue on Formal Ontology in Conceptual Analysis and Knowledge Representation. Eds, Guarino, N. \& Poli , R., 1993

[21] Guarino, N., and Poli, R., Eds. Towards Principles for the Design of Ontologies Used for Knowledge Sharing (Deventer, The Netherlands, 1993), Kluwer Academic Publishers.

[22] Guan-yu, L.., Li-ning, L. ,Shi-peng, L. Design and realization of case-based ontology reasoning. In 4th International Conference on Wireless Communications, Networking and Mobile Computing, 2008. WiCOM '08. (2008).

[23] Hwang M., Kong H., Baek S., Hwang K., Kim P. The techniques for the ontology-based information retrieval. In The 9th International Conference on Advanced Communication Technology (Gangwon-Do, South Korea, 2007), IEEE.

[24] Juan A. Recio-García, Belén Díaz-Agudo, Pedro A. onzálezCalero, and Antonio Ontology based CBR with jCOLIBRI, Applications and Innovations in Intelligent Systems XIV.Proceedings of AI-2006, the Twenty-sixth SGAI International Conference on Innovative Techniques and Applications of Artificial Intelligence, pages 149-162, Cambridge, United Kingdom, December 2006. Springer

[25] Lenz, M., Bartsch-Sporl, B., Burkhard, H., and Wess, S. Case-Based Reasoning, Technology - From Foundation to Applications,Lecture Notes in Artificial Intelligence 1400, Springer Verlag, 1998

[26] C. Masolo : Ontology driven Information retrieval: Stato dell'arte. Report of the IKF (Information and Knowledge Fusion) Eureka Project EU2235. LADSEB-Cnr, Padova (I), 2004.

[27] Minor, M., and Staab, S., Eds. Architecture-Based Integration of CBR-Components into KM-Systems (2002), vol. 10 of LNI, GI.

[28] G. Pasi, "Flexible Information Retrieval: some research trends", Mathware and Soft Computing, Vol IX, number 9-1, pp. 107-121, 2002.

[29] Patterson, D. W., Rooney, N., and Galushka, M. Efficient similarity determination and case construction techniques for case-based reasoning. Lecture Notes in Computer Science 2416 (2002), 292-297.
[30] Ramirez, C. Case-based Reasoning Applied to Information Retrieval. In IEE Coloquium on Case-Based Reasoning: Prospects for Application (London, Feb. 1995), IEEE.

[31] Ralph Bergman, Martin Schaaf, On the Relation between Structural Case-Based Reasoning and Ontology-Based Knowledge management, In Proc. of German Workshop On Experience Management, April, 2003.

[32] Rissland, E. L., and Daniels, J. J. Using CBR to drive IR. In IJCAI (1995), pp. 400-407.

[33] Rocchio, J. J. Relevance feedback in information retrieval. In The Smart retrieval system - experiments in automatic document processing, G. Salton, Ed. Englewood Cliffs, NJ: Prentice-Hall, 1971, pp. 313-323.

[34] Rose, D., L. D. Understanding user goals in web search. In 13th International Conference on World Wide Web (USA, 2004), ACM Press, pp. 13-19.

[35] Salton, G., and Buckley, C. Term-weighting approaches in automatic text retrieval. Information Processing and Management 24, 5 (1988), 513-523.

[36] Salton, G., and McGill, M. J. Introduction to Modern Information Retrieval. McGraw Hill, 1983.

[37] Sánchez D. et Moreno A. Creating ontologies from Web documents. Proceedings of the Setè Congrés Català d'Intel.ligència Artificial, IOS Press 113, 2004.

[38] Vakkari, P. \& Järvelin, K. Explanation in Information Seeking and Retrieval. In: Spink, A. \& Cole, C. (ed.), New Directions in Cognitive Information Retrieval. Berlin: Springer, The Information Retrieval Series, Vol.19, 2005, pp. 113-138.

[39] Vehvilainen A., Olli Alm, E. H. Combining case-based reasoning and semantic indexing in a question-answer service. In 1st Asian Semantic Web Conference (2006).

[40] Wei Wang, Payam M. Barnaghi, Andrzej Bargiela, "Search with Meanings:An Overview of Semantic Search Systems", International journal of Communications of SIWN, Vol. 3, June 2008, pp. 76-82.

[41] L. Kerschberg, H. Jeong, Y.U. Song, W. Kim, A Case-Based Framework for Collaborative Semantic Search in Knowledge Sifter. 2007 International Conference on Case-Based Reasoning (ICCBR 2007), LNAI 4626, R.O. Weber and M.M. Richter (Eds.) Belfast, UK, August 13-16, 2007, pp. 16-30.

[42] Bradley, K., Smyth, B.: An Architecture for Case-Based Personalised Search. In: Funk, P., Gonz'alez Calero, P.A (eds.) ECCBR 2004. LNCS (LNAI), vol. 3155, pp.518-532. Springer, Heidelberg , 2004.

[43] MillerG.A. : WORDNET: A Lexical Database for English, Communications of ACM, 1995, pp. 39-41.

[44] D. Leake and A. Kinley and D. WilsonCase-Based luppercaseCBR: Capturing and Reusing Reasoning About Case Adaptation, International Journal of Expert Systems, 10(2), pp. 197-213, 1997. 